\title{
Como os livros-texto de Odontopediatria e Ortodontia abordam os temas aleitamento materno e alimentação complementar?
}

\author{
Lucimeire de Sales Magalhães Brockveld*; Sonia Isoyama Venâncio** \\ * Cirurgiã-dentista, Doutora, Professora Curso de Medicina, \\ Universidade Cidade de São Paulo \\ ** Médica pediatra, Diretora técnica do Instituto de Saúde da \\ Secretaria de Saúde do Estado de São Paulo
}

Recebido em 04/07/2019. Aprovado em 10/04/2020.

\begin{abstract}
RESUMO
$\mathrm{O}$ aleitamento materno (AM) e a alimentação complementar (AC) contribuem para o desenvolvimento do sistema estomatognático e evitam doenças bucais como a cárie e maloclusão. Este artigo apresenta um estudo qualitativo de análise documental para verificar as informações sobre AM e AC em quatro livros de referência para a graduação em Odontologia no Brasil, sendo dois livros de Odontopediatria e dois de Ortodontia. Os livros foram escolhidos por indicação na bibliografia destas duas disciplinas em seis cursos de Odontologia do estado de São Paulo. A pesquisa demonstrou que as informações estão em maior quantidade e profundidade nos livros de Odontopediatria e algumas necessitam de atualização. Conclui-se que a literatura estudada, de apoio ao aprendizado do estudante de Odontologia apresenta conteúdo útil e pertinente e serve como disparador para novas buscas. Lacunas importantes foram identificadas nos livros de Ortodontia analisados em relação aos benefícios do AM e AC que podem contribuir para a prevenção de doenças bucais. Os livros de Odontopediatria trazem conteúdo mais completo, sendo que o livro publicado em 2017 é também o mais atualizado, embora alguns aspectos necessitem ainda de alinhamento com as novas evidências científicas e com as orientações dos guias alimentares nacionais.

Descritores: Educação em Odontologia. Aleitamento Materno. Fenômenos Fisiológicos da Nutrição do Lactente. Promoção de Saúde.
\end{abstract}




\section{INTRODUÇÃO}

A amamentação melhora a sobrevivência e a saúde das crianças, conferindo proteção contra infecções e maloclusão, reduzindo a ocorrência de sobrepeso e contribuindo para o desenvolvimento do capital humano ${ }^{1}$. A Organização Mundial de Saúde (OMS) ${ }^{2}$ e o Ministério da Saúde $(\mathrm{MS})^{3}$ preconizam o aleitamento materno (AM) exclusivo até 6 meses de vida, quando a criança deve começar a receber alimentação (AC) complementar, e continuado até os dois anos de vida ou mais.

O AM é um importante fator para a maturação e o desenvolvimento da musculatura facial, promovendo o crescimento e desenvolvimento do sistema estomatognático (SE), preservando suas funções vitais. A mastigação, iniciada a partir do surgimento dos primeiros dentes decíduos, requer alimentos duros, secos e fibrosos para permitir o correto desenvolvimento do $\mathrm{SE}^{4}$.Outro fator de modificação do trabalho muscular efetuado pelo AM é o uso da mamadeira e outros bicos ${ }^{5}$.

Apesar dos benefícios do AM e da expansão desta prática no Brasil, os indicadores ainda estão aquém das recomendações da $\mathrm{OMS}^{6,7}$. Em relação à AC, os dados da Pesquisa Nacional de Demografia e Saúde (PNDS-2006) evidenciam alta frequência de crianças ingerindo alimentos de baixo valor nutricional, e baixa frequência de crianças consumindo frutas, carnes e verduras ${ }^{8}$.

É fundamental que as mães, os cuidadores e os familiares recebam orientações adequadas e sejam apoiados para a prática do AM pelos diversos profissionais da área da saúde. Consequentemente, a formação profissional inadequada é uma barreira para as transformações desejadas. Em todos os perfis de profissionais de saúde existem lacunas substanciais no conhecimento e nas habilidades para apoiar a amamentação?
Considerando as competências e habilidades elencadas nas Diretrizes Curriculares Nacionais (DCN) ${ }^{10,11}$ dos cursos de graduação em Odontologia é preciso qualificar a formação deste profissional no que diz respeito à prevenção de doenças bucais atribuídas ao AM e AC oferecendo subsídios para que possa atuar nas ações de apoio, promoção e proteção desta prática, que pode evitar desfechos negativos em crianças de todas faixas de renda.

Diante do exposto, o objetivo deste estudo é verificar como os livros-texto de Odontopediatria e Ortodontia abordam os temas $\mathrm{AM} \mathrm{e} \mathrm{AC}$.

\section{METODOLOGIA}

Este artigo apresenta parte dos resultados da tese de doutorado da autora principal, que avaliou a graduação em Odontologia sob o referencial teórico das $\mathrm{DCN}^{10,11}$, com foco no ensino do AM e AC.

É um estudo qualitativo utilizando a análise documental. O uso de documentos em pesquisa possibilita o resgate de informações tornando possível ampliar o entendimento de objetos cuja compreensão necessita de contextualização histórica e sociocultural ${ }^{12}$. Segundo Bravo ${ }^{13}$, são documentos todas as realizações produzidas pelo homem que se mostram como indícios de sua ação e que podem revelar suas ideias, opiniões e formas de atuar e viver.

Para selecionar os documentos do estudo, foram utilizadas as ementas de seis cursos de Odontologia, sendo três de instituições públicas (Faculdade de Odontologia de Araraquara da Universidade Paulista "Júlio de Mesquita Filho"/UNESP, Faculdade de Odontologia da Universidade de São Paulo/FOUSP e Faculdade de Odontologia de Piracicaba da Universidade Estadual de Campinas/FOP-UNICAMP) e três de instituições privadas (Universidade Metodista de São Paulo /UMESP, Universidade 
Paulista/UNIP e Universidade Cidade de São Paulo/ UNICID). A escolha destes cursos baseou-se na avaliação anual do ensino superior do Brasil do jornal Folha de São Paulo ${ }^{14}$. No ranking, estão classificadas as 195 universidades brasileiras, públicas e privadas, a partir de cinco indicadores: pesquisa, internacionalização, inovação, ensino e mercado.

Os livros mais mencionados foram: Ortodontia $^{15}$ (livro I), Ortodontia - Diagnóstico e Planejamento Clínico $^{16}$ (livro II), Odontopediatria $^{17}$ (livro III) e Odontopediatria na Primeira Infância - uma visão multidisciplinar $^{18}$ (livro IV).

Todos os quatro livros foram lidos integralmente pela pesquisadora principal. A análise foi baseada em buscar conteúdos relacionados aos temas "Aleitamento materno como a escolha alimentar mais saudável"; "Aleitamento materno exclusivo nos 6 primeiros meses de vida"; "Aleitamento materno continuado por 2 anos ou mais"; "Aleitamento materno como promotor do desenvolvimento do SE"; "Alimentação complementar como indutora do desenvolvimento do SE"; e "Maus hábitos orais e o desenvolvimento de doenças bucais".

\section{RESULTADOS E DISCUSSÃO}

Os quatro livros analisados são clássicos da literatura odontológica e como tal, são referências para estudantes de graduação, pósgraduação e profissionais. Foram utilizadas as edições mais recentes de cada livro, com datas variando de 1991 a 2017.

No livro $\mathrm{III}^{17}$ há um tópico inteiro destinado ao AM no capítulo 27, mostrando seus benefícios para a saúde da criança e enfatizando a importância de medidas que promovam esta prática. $\mathrm{O}$ livro $\mathrm{IV}^{18}$ é o único livro que tem um capítulo inteiro para o tema, além de abordá-lo em vários outros capítulos. É o livro onde mais vezes se aborda o AM e a AC.
As recomendações sobre o AM ser a escolha alimentar mais saudável, ser exclusivo por seis meses e continuado por dois anos ou mais estão presentes nos livros de Odontopediatria. Os livros de Ortodontia não mencionam nada sobre estas orientações, conforme mostra a figura 1 .

A orientação correta para o AM até dois anos ou mais está presente nos capítulos 6,9 , e 10 do livro $\mathrm{IV}^{18}$. No entanto, o capítulo 11 , apresenta a informação sobre a oportunidade fisiológica para retirada do hábito de sucção: "...nessa fase (7-8 meses de vida), o desaparecimento da chupeta e a passagem do peito para copos se faz de maneira tranquila".

O desenvolvimento do SE e a fisiologia da amamentação são descritos detalhadamente nos livros de Odontopediatria, aparecendo de forma mais sintética nos livros de Ortodontia. Os textos dos livros de Odontopediatria apresentam, também, os benefícios do AM para a saúde da criança, a formação do vínculo mãe-bebê, as vantagens para a saúde da mãe e os benefícios para a sociedade como um todo.

Sobre o tema da introdução da AC adequada para continuar induzindo $\mathrm{O}$ desenvolvimento das estruturas faciais, os livros de Odontopediatria abordam a necessidade de orientação aos pais e detalham a importância de estimular corretamente a mastigação. $\mathrm{O}$ livro $\mathrm{II}^{16}$ menciona brevemente o desenvolvimento da mastigação pelo consumo de comidas mais sólidas, conforme a figura 2 .

As evidências trazidas nos livros explicitam que a amamentação é fator decisivo e primordial para a correta maturação e crescimento das estruturas, mantendo-as aptas para exercerem o desenvolvimento da musculatura orofacial, que guiará e estimulará o desenvolvimento das funções fisiológicas, garantindo não só a sobrevivência, mas também melhor qualidade de vida ${ }^{4}$. 
Figura 1. Exemplos de citações encontradas sobre as recomendações quanto ao AM

\begin{tabular}{|c|c|c|c|c|}
\hline & Livro $\mathbf{I}^{15}$ & Livro II $^{16}$ & Livro III $^{17}$ & Livro IV $^{18}$ \\
\hline 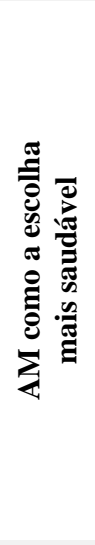 & Ausente & Ausente & $\begin{array}{l}\text { "Inúmeros estudos comprovam os } \\
\text { benefícios do aleitamento } \\
\text { materno sobre a saúde da } \\
\text { criança, enfatizandorr a } \\
\text { importância de medidas que } \\
\text { possam promover o aleitamento } \\
\text { materno." (Capítulo 27) }\end{array}$ & $\begin{array}{l}\text { "O aleitamento materno é o vínculo afetivo mais } \\
\text { positivo na relação mãe-filho, já que não só garante um } \\
\text { desenvolvimento psicoemocional adequado, mas } \\
\text { impacta os indicadores de saúde materno-infantil ao } \\
\text { longo da vida." (Capitulo 9) } \\
\text { "Nada é melhor para a criança do que a alimentação no } \\
\text { peito. O aleitamento natural é ideal para o recém- } \\
\text { nascido porque, além de o leite materno ser modulado } \\
\text { para o bebê suprindo suas necessidades até o } 6^{\circ} \text { mês de } \\
\text { vida, também fornece anticorpos contra várias doenças, } \\
\text { tem um papel na maturação gastrointestinal, no } \\
\text { aumento do desempenho neurocomportamental, } \\
\text { promove melhor desenvolvimento cognitivo e } \\
\text { psicomotor e menos incidência de reinternação } \\
\text { hospitalar." (Capítulo 10) }\end{array}$ \\
\hline 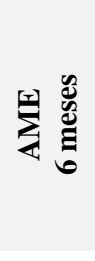 & Ausente & Ausente & $\begin{array}{l}\text { "O aleitamento materno } \\
\text { exclusivo até } 6 \text { meses de idade da } \\
\text { criança deve ser incentivado, não } \\
\text { sendo necessário qualquer } \\
\text { complementação alimentar, nem } \\
\text { mesmo chá ou água neste } \\
\text { período." (Capítulo 27) }\end{array}$ & $\begin{array}{l}\text { "O Ministério da Saúde do Brasil (MS) segue as } \\
\text { diretrizes da Organização Mundial de Saúde (OMS), } \\
\text { preconizando o aleitamento materno exclusivo (AME) } \\
\text { até os } 6 \text { meses, estendido até os } 2 \text { anos de vida. Tal } \\
\text { prática tem importância fundamental na sobrevivência, } \\
\text { crescimento e desenvolvimento dos lactentes." } \\
\text { (Capítulos } 6,9,10,11)\end{array}$ \\
\hline 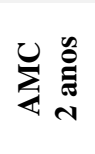 & Ausente & Ausente & Ausente & $\begin{array}{l}\text { "A OMS e o MS do Brasil preconizam a continuidade do } \\
\text { aleitamento materno até os } 2 \text { anos de idade em paralelo } \\
\text { à introdução de alimentação complementar balanceada } \\
\text { e equilibrada." (Capítulo } 6,9,10 \text { ) }\end{array}$ \\
\hline
\end{tabular}

AM: Aleitamento Materno; AME: Aleitamento Materno Exclusivo; AMC: Aleitamento Materno Continuado.

Figura 2. Citações encontradas nos livros sobre AM e AC no desenvolvimento do SE

\begin{tabular}{|c|c|c|}
\hline & $\begin{array}{c}\text { AM como promotor } \\
\text { do desenvolvimento do } \mathrm{SE}\end{array}$ & AC como indutora do desenvolvimento do SE \\
\hline Livro $I^{15}$ & $\begin{array}{l}\text { "A deglutição infantil, função essencial no recém- } \\
\text { nascido, está intimamente associada com a } \\
\text { amamentação e ambos estão bem desenvolvidos por } \\
\text { volta da } 32^{a} \text { semana de vida intra-uterina". } \\
\text { (Capítulo 5) }\end{array}$ & Ausente \\
\hline Livro II $^{16}$ & $\begin{array}{l}\text { "[...]quando a sucção é feita pelo seio materno, } \\
\text { promove um maior desenvolvimento e maturação } \\
\text { dos músculos periorais." (Capítulo 13) }\end{array}$ & $\begin{array}{l}\text { "A transição da deglutição infantil para a madura ocorre após alguns } \\
\text { meses, ajudada pela maturação de elementos neuromusculares, [...] o } \\
\text { instintivo desejo de mastigar, a necessidade de utilizar comidas mais } \\
\text { sólidas, o desenvolvimento dentário etc." (Capítulo 5) }\end{array}$ \\
\hline Livro III $^{17}$ & $\begin{array}{l}\text { "Os movimentos musculares para a ordenha do peito } \\
\text { são intensos e preparam os músculos para que } \\
\text { exerçam uma boa função mastigatória no futuro." } \\
\text { (Capítulo 5) }\end{array}$ & $\begin{array}{l}\text { "O padrão da alimentação infantil vem se modificando com a } \\
\text { substituição de grande parte dos alimentos naturais pelos } \\
\text { industrializados [...] que não estimulam a mastigação, fundamental } \\
\text { para o equilibrio do desenvolvimento oclusal e a prevenção da cárie } \\
\text { dentária." (Capítulo 27) }\end{array}$ \\
\hline Livro IV ${ }^{18}$ & $\begin{array}{l}\text { “A sucção nutritiva sobretudo a natural, favorece o } \\
\text { crescimento e o desenvolvimento da mandíbula e dos } \\
\text { maxilares, bem como dos músculos labiais e linguais, } \\
\text { a manutenção do modo de respiração nasal, o } \\
\text { equilíbrio oclusal e o remodelamento das } \\
\text { articulações temporomandibulares" (Capítulo 3) } \\
\text { "A amamentação é fundamental, pois promoverá a } \\
\text { vedação anterior do espaço oral quando a criança } \\
\text { faz o vedamento labial para a apreensão da mama, } \\
\text { com os lábios evertidos, estimulando a atividade dos } \\
\text { músculos periorais." (Capítulo 8) }\end{array}$ & $\begin{array}{l}\text { "Quando a transição alimentar é realizada de forma correta na } \\
\text { primeira infância, com apresentação ordenada de consistências e } \\
\text { texturas alimentares diferentes, o desenvolvimento equilibrado das } \\
\text { estruturas e funções orofaciais bem como da saúde em geral são } \\
\text { favorecidos" Capítulo } 3 \\
\text { "A evolução inadequada da alimentação no primeiro ano de vida é } \\
\text { capaz de promover alterações no estado nutricional e na saúde bucal } \\
\text { por comprometimento do desenvolvimento do SE." (Capítulo 6) } \\
\text { "A introdução de alimentos sólidos na dieta necessita de orientações } \\
\text { específicas da área odontológica devido à importância do } \\
\text { estabelecimento da função mastigatória, através do incentivo aos } \\
\text { alimentos mais consistentes, fibrosos e secos." (Capitulo 14) }\end{array}$ \\
\hline
\end{tabular}

AM: Aleitamento Materno; SE: Sistema Estomatognático; AC: Alimentação Complementar. 
A introdução correta de alimentos na dieta do bebê a partir dos seis meses de idade contribui para a sua saúde e continua estimulando o desenvolvimento adequado das estruturas bucais dependendo da qualidade e da textura dos alimentos oferecidos.

A preocupação com a ingestão de alimentos industrializados e ultraprocessados citada no livro III $^{19}$ se faz porque a ingestão destes alimentos tendem a limitar o consumo de alimentos in natura ou minimamente processados, além de estar ligado ao consumo excessivo de calorias, sal e gorduras ${ }^{17}$.

Os livros relacionam fortemente $\mathrm{o}$ desenvolvimento de hábitos orais com alterações de todos os tipos no desenvolvimento das estruturas bucais. Os hábitos são de natureza complexa e caracterizados pela constância e repetição, tornando-se inconscientes. A falta de amamentação com a introdução de mamadeiras é uma das causas da instalação de hábitos orais, provocando alterações estruturais e funcionais, levando a maloclusão ${ }^{4}$. Dados das Pesquisas Nacionais de Prevalência do AM mostram que o uso da chupeta é um fator fortemente associado à interrupção precoce do AM exclusivo no Brasil ${ }^{20}$.

A orientação do uso de bicos ortodônticos aparece no capítulo 13 do livro II $^{16}$. Bishara et al. ${ }^{21}$ afirmam que poucos dados científicos comprovam substancialmente a existência de alguma vantagem no uso de bico ortodôntico. O resultado de uma revisão sistemática expressou que não há diferenças significativas entre os bicos ortodôntico e convencional quanto às implicações no $\mathrm{SE}$, embora as pesquisadoras indiquem a necessidade de mais estudos sobre o assunto ${ }^{22}$. As evidências mostram que o uso de chupeta de qualquer tipo é a causa mais associada às baixas taxas de $\mathrm{AM}^{23}$.

Nos textos analisados, encontram-se mensagens que podem deixar o estudante (principalmente de graduação) inseguro na orientação do aleitamento materno, como mostra a figura 3 .

Figura 3. Exemplo de citações encontradas nos livros de desestímulo ao AM

\begin{tabular}{|l|l|}
\hline Livro II' & $\begin{array}{l}\text { "No entanto, por razões várias, dificilmente a mãe hoje em dia reúne condições para a amamentação. " (Capítulo } \\
13)\end{array}$ \\
\hline Livro III'17 & $\begin{array}{l}\text { "Alguns autores afirmam que a necessidade e o reflexo de sucção da criança persistem até seu segundo ano de } \\
\text { vida. No entanto, a partir do sexto mês de vida, é aconselhável o desmame gradativo". (Capítulo 27) }\end{array}$ \\
\hline
\end{tabular}

As mulheres devem ser informadas sobre as vantagens do AM exclusivo e das desvantagens da introdução precoce de outros alimentos e receber apoio da família, da comunidade, do trabalho, de profissionais de saúde preparados e da sociedade em geral. Ações conjuntas e políticas públicas que respeitem o direito da mulher e da criança, favorecem que a mãe reúna condições para a amamentação.

\section{Informações que necessitam atualização}

Os livros didáticos periodicamente revisam suas edições, mas a evolução das pesquisas e das evidências científicas podem deixar algumas informações desatualizadas. Foram encontradas algumas orientações que necessitam atualização de acordo com as novas evidências.

Sobre o preparo dos alimentos, por ocasião da introdução alimentar, o livro III $^{17}$ (capítulo 27) indica que a sopa deva ser passada em peneiras. A revisão do Guia Alimentar para crianças brasileiras menores de dois anos ${ }^{24}$ orienta a introdução aos seis meses de idade e a não oferecer preparações líquidas e não usar liquidificador, mixer ou peneira. O recomendável é a criança receber a comida amassada com garfo e depois evoluir para alimentos picados em pedaços pequenos, raspados ou desfiados. 
Manobras para aumentar e fortalecer os mamilos durante a gestação, como orientado no capítulo 40 do livro III $^{17}$, não são recomendadas como no passado pois a própria gravidez se encarrega de proporcionar condições para a amamentação.

Para a prevenção de fissuras, o Caderno de Atenção Básica $(\mathrm{CAB}) 23^{3}$ propõe medidas como: amamentação com técnica adequada (principalmente a pega), manter os mamilos secos, não usar produtos que retirem a proteção natural do mamilo como sabões e amamentação em livre demanda. O capítulo 6 do livro $\mathrm{IV}^{18}$ traz uma informação adequada quando preconiza que a criança deva sugar livremente o seio materno, sem a preocupação com o tempo da mamada, mas no capítulo 5 enfatiza que cada mamada deve ter o tempo exato de 15 minutos de cada lado. Este mesmo capítulo diz que a livre demanda impede que o bebê sugue forte e afirma que quanto mais forte a sucção, mais adequado é o leite. Segundo a orientação do CAB 23, o tempo de permanência na mama em cada mamada não deve ser fixado, considerando que o importante é esvaziar adequadamente a mama e isto varia para cada dupla mãe/bebê.

Um assunto controverso que aparece ao longo do livro $\mathrm{IV}^{18}$ é sobre limpeza da cavidade oral do bebê antes da erupção do primeiro dente. Nos capítulos 10, 16 e 18 oferecem a orientação de limpeza após a mamada nos recém-nascidos com gaze embebida em soro fisiológico ou com solução de higiene bucal específica. No mesmo capítulo 18, diz parecer desnecessária a limpeza no bebê edêntulo pois sua cavidade não apresenta nichos para a retenção de microrganismos associados à cárie e doença periodontal. O leite materno forma uma camada de imunoglobulinas em toda mucosa bucal que protege esta via de entrada de uma série de doenças, e sua retirada com gaze/fralda embebida em soro fisiológico/água filtrada ou fervida diminui a proteção delegada ao beb $\hat{e}^{25}$.
A análise dos conteúdos dos livros indicados aos estudantes de graduação no curso de Odontologia nas disciplinas de Odontopediatria e Ortodontia colabora na compreensão do que se oferece de apoio aos estudantes em complemento às aulas ministradas, em relação aos temas tratados.

A intenção do estudo foi colaborar para que cada vez mais os livros didáticos e os artigos científicos destaquem a importância do AM e da AC saudável para uma melhor saúde geral e bucal das crianças e famílias e, principalmente, conscientizar futuros e atuais cirurgiões-dentistas, sobre sua responsabilidade como profissional de saúde, na correta informação e no melhor manejo nas práticas cotidianas.

Considera-se que há lacunas a serem preenchidas principalmente pelos livros de Ortodontia, mudando do paradigma restaurador para o da promoção da saúde e prevenção de doenças bucais. Deve-se buscar cada vez mais a atualização de acordo com as evidências científicas e as orientações ministeriais.

Esta é a primeira análise dessa natureza relacionada aos assuntos pesquisados e traz contribuições para o aprimoramento dos livros de Ortodontia e Odontopediatria utilizados por estudantes de Odontologia.

Uma limitação deste estudo foi a seleção dos livros para revisão, que se baseou nas indicações das disciplinas. Se reconhece que pode haver informações mais atualizadas e precisas em outros livros referenciados aos estudantes. Novas pesquisas devem ampliar o conhecimento $\mathrm{e}$ qualificar a discussão sobre o tema.

\section{CONCLUSÃO}

Conclui-se que a literatura estudada, que apoia o aprendizado do estudante de Odontologia, apresenta conteúdo útil e pertinente e serve como disparador para novas buscas. Lacunas importantes foram identificadas nos livros de Ortodontia analisados em relação aos benefícios do AM e AC 
que podem contribuir para a prevenção de doenças bucais. Os livros de Odontopediatria trazem conteúdo mais completo, sendo que o livro publicado em 2017 é também o mais atualizado, embora alguns aspectos necessitem ainda de alinhamento com as novas evidências científicas e com as orientações dos guias alimentares nacionais.

\section{ABSTRACT \\ How do Pediatric Dentistry and Orthodontics textbooks address the themes of breastfeeding and complementary feeding?}

Breastfeeding (BF) and complementary feeding (CF) contribute to the development of the stomatognathic system and prevent oral diseases such as dental caries and malocclusion. This article reports a qualitative document analysis study to check information about BF and CF in four reference books used in Dentistry undergraduate programs in Brazil, namely two Pediatric Dentistry books and two Orthodontics ones. The books were chosen because they are included as course readings in the bibliography of the two courses in six Dentistry undergraduate programs in the state of São Paulo. This research showed that in the Pediatric Dentistry books, information is found in a greater amount and is more detailed, and some of this information needs updating. In conclusion, the study literature, which supports the learning of Dentistry students, presents useful and relevant content and stimulates the search for further information. Important gaps were found in the target Orthodontics books as regards the benefits of $\mathrm{BF}$ and $\mathrm{CF}$ that can aid the prevention of oral diseases. The contents in the Pediatric Dentistry books are more complete, and the book published in 2017 is also the most updated, although some aspects still need to be aligned with new scientific evidence and with the advice of the national dietary guidelines.

Descriptors: Education, Dental. Breast Feeding. Infant Nutritional Physiological Phenomena. Health Promotion.

\section{REFERÊNCIAS}

1. Victora CG, Bahl R, Barros AJ, França GV, Horton S, Krasevec J, et al. Breastfeeding in the 21st century: epidemiology, mechanisms, and lifelong effect. Lancet. 2016; 387(10017):475-90.

2. Kramer MS, Kakuma R. The optimal duration of exclusive breastfeeding: a systematic review. Geneva: WHO; 2002 [Acesso em 15/05/19]. Disponível em: https://www.who.int/nutrition/publications/ optimal_duration_of_exc_bfeeding_review eng.pdf

3. Brasil. Ministério da Saúde. Secretaria de Atenção à Saúde. Departamento de Atenção Básica. Saúde da criança: aleitamento materno e alimentação complementar. $2 \mathrm{a}$ ed. Brasília: MS; 2015. (Caderno de Atenção Básica; n. 23).

4. Carvalho GD. SOS Respirador Bucal, uma visão funcional e clínica da amamentação. São Paulo: Lovise; 2003.

5. Oliveira MMT, Amorim VCSA. Aleitamento materno: promoção de saúde na infância. Odontol Clin-Cient. 2005, 4(1):4956.

6. Venancio SI, Monteiro CA. A tendência da prática da amamentação no Brasil nas décadas de 70 e 80. Rev Bras Epidemiol. 1998; 1(1):40-9.

7. Venancio SI, Saldiva SRDM, Monteiro CA. Tendência secular da amamentação no Brasil. Rev Saúde Pública. 2013; 47(6):1205-8.

8. Boccolini CS, Boccolini PMM, Monteiro FR, Venancio SI, Giugliani ERJ. Tendência de indicadores do aleitamento materno no Brasil em três décadas. Rev Saude Publica [online]. 2017 [Acesso em 16/03/19]. Disponível em: http://www.scielo.br/pdf/ rsp/v51/pt 0034-8910-rsp-S1518-8787201 7051000029.pdf.

9. Leviniene G, Petrauskiene A, Tamuleviciene E, Kudzyte J, Labanauskas L. The evaluation of knowledge and activities of primary health care professionals in promoting breast-feeding. Medicina (Kaunas). 2009; 45(3):238-47. 
10. Brasil. Ministério da Educação. Resolução CNE/CES no 3 de 19 de fevereiro de 2002. Institui as Diretrizes Curriculares Nacionais do Curso de Graduação em Odontologia. Diário Oficial da União, Brasília (DF). 2002 4 mar.; Seção 1:10.

11. Resolução CNE/CES n ${ }^{\circ} 803$ de 5 de dezembro de 2018. Revisão das Diretrizes Curriculares Nacionais do Curso de Graduação de Odontologia. Aguardando homologação do Ministério da Educação. [Acesso em 23/12/19]. Disponível em: http://portal.mec.gov.br/docman/abril-2019pdf/111231-pces803-18/file.

12. Sá-Silva JR, Almeida CD, Guindani JF. Pesquisa documental: pistas teóricas e metodológicas. Rev Bras Hist Ciênc Soc [internet]. Jul 2009 [Acesso em 16/03/19]. Disponível em: https://www.rbhcs.com/ rbhcs/article/view/6/pdf.

13. Bravo RS. Técnicas de investigação social: teoria e ejercicios. 7a ed rev. Madrid: Paraninfo; 1991.

14. Ranking Universitário Folha de São Paulo, 2017. [Acesso em 30/06/17]. Disponível em: https://ruf.folha.uol.com.br/2017/rankin gde-cursos/odontologia/.

15. Moyers RE. Ortodontia. 4a ed. Rio de Janeiro: Guanabara Koogan; 1991.

16. Ferreira FV. Ortodontia: diagnóstico e planejamento clínico. 7a ed. São Paulo: Artes Médicas; 2008

17. Guedes-Pinto AC. Odontopediatria. 8a ed. São Paulo: Santos; 2010.

18. Corrêa MSNP. Odontopediatria na primeira infância - uma visão multidisciplinar. 3a ed. São Paulo: Quintessence Editora; 2017.
19. Brasil. Ministério da Saúde. Secretaria de Atenção à Saúde. Departamento de Atenção Básica. Guia alimentar para a população brasileira. 2a ed. Brasília: MS; 2014.

20. Buccini GS, Pérez-Escamilla R, Venancio SI. Pacifier use and exclusive breastfeeding in Brazil. J Hum Lact. 2016 Aug; 32(3):NP52-60.

21. Bishara SE, Nowak AJ, Kohout FJ, Heckert DA, Hogan MM. Influence of feeding and non-nutritive sucking methods on the development of the dental arches: longitudinal study of the first 18 months of life. Pediatr Dent. 1987; 9(1):13-21.

22. Corrêa CC, Bueno MRS, Lauris JRP, Berretin-Felix G. Interferência dos bicos ortodônticos e convencionais no sistema estomatognático: revisão sistemática. CoDAS. 2016; 28(2):182-9

23. Buccini GDS, Pérez-Escamilla R, Paulino LM, Araújo CL, Venancio SI. Pacifier use and interruption of exclusive breastfeeding: systematic review and meta-analysis. Matern Child Nutr [internet]. 2017 Jul [Acesso em 15/05/19]. Disponível em: https://doi.org/10.1111/mcn.12384.

24. Brasil. Ministério da Saúde. Secretaria de Atenção Primária à Saúde. Departamento de Promoção da Saúde. Guia alimentar para crianças brasileiras menores de 2 anos. Brasília: Ministério da Saúde, 2019.

25. Burt B.; Eklund SA. Dental Caries. In: Dentistry dental Practice, and the Community. 4.ed. Philadelphia: Saunders, 1992. p.90-112.

\section{Correspondência para:}

Lucimeire de Sales Magalhães Brockveld e-mail: lucimeirebrockveld@gmail.com Rua Prof. Monjardino, 130 - 84B Vila Sônia 05625-160 São Paulo/SP 